Hart, P. D'A. \& Rees, R. J. W. (1954). J. gen. Microbiol. 10, 150-159.

\title{
Changes in Morphology of an Avirulent Strain of Myco- bacterium tuberculosis under the influence of Non-ionic Surface-active Agents
}

\author{
BY P. D'ARCY HART AND R. J. W. REES \\ The National Institute for Medical Research, Mill Hill, London, N.W. 7
}

SUMMARY : When the avirulent strain of Mycobacterium tuberculosis H37 Ra was repeatedly subcultured in a liquid medium containing low concentrations of 'Triton A 20' or other chemically similar non-ionic surface-active polyoxyethylene ethers, the morphology changed from the amorphous growth pattern characteristic of avirulent tubercle bacilli to the formation of microscopic 'cords' similar to those shown by virulent strains such as H37Rv. Once developed, the cord-forming property of H37 Ra persisted when cultures were transferred to liquid medium devoid of surface-active agent, even when such transfer was made after passage through mice; after further subcultures, however, a tendency to reversion was apparent. No difference in virulence was detected between cord-forming H37 Ra and the unmodified strain when tested in mice.

Previous work had indicated that a lower member of the polyoxyethylene ether series, the dimer ' $D 4$ ', inhibited amorphous-growing avirulent tubercle bacilli, including $\mathrm{H37} \mathrm{Ra}$, but not the growth of naturally cord-forming strains, exemplified by the virulent $\mathrm{H37Rv}$ and attenuated strains derived from it. The growth of cord-forming avirulent organisms, developed from $\mathrm{H3} 3 \mathrm{Ra}$ in the present experiments, was also found to be relatively unaffected by $\mathrm{D} 4$.

During an investigation of the properties of an attenuated strain of Mycobacterium tuberculosis (VAL, see Rees \& Hart, 1952), derived from the virulent human variant $\mathrm{H} 37 \mathrm{Rv}$, we noted that virulence could be partly restored by repeated subculture in a medium containing the commercial non-ionic surfaceactive agent 'Triton A 20' (Hart \& Rees, unpublished). Tests were therefore made of the behaviour of some standard avirulent strains of $\boldsymbol{M}$. tuberculosis after similar treatment. The morphology was strikingly changed in one such strain, although no significant virulence was induced. It is a characteristic of virulent tubercle bacilli to form microscopic 'cords' when grown in certain liquid media, whereas avirulent strains grow amorphously. The change in morphology here to be described was away from an amorphous, and towards a cord, pattern of growth.

\section{MATERIALS AND METHODS}

Surface-active agents. 'Triton A20' was made by Rohm and Haas Co., Philadelphia, and supplied by Charles Lennig and Co., Ltd., London; it is stated to be a $25 \%$ solution of 'Triton WR 1339', a polymeric $p$-isooctyl polyoxyethylene phenol (see Rees \& Hart, 1952; Friedman \& Byers, 1953). 'Tween 80', a polyoxyethylene derivative of sorbitan mono-oleate, was made by Atlas Powder Co., Wilmington, Delaware. The other agents were non-ionic surface-active polyoxyethylene ethers which had been synthesized by our 
colleagues Drs J. W. Cornforth and J. A. Stock in the course of studies following the observation that certain products of this type (including 'Triton A 20') suppressed the development of experimental tuberculosis in mice and guinea-pigs (Cornforth, Hart, Rees \& Stock, 1951; Hart, Long \& Rees, 1952; Rees, 1953). The gęneral formula of this series has been given elsewhere (see Rees \& Hart, 1952). The products used here were the dimer D4 (the ether of 2, 2'-dihydroxy-5, 5'-di-tert-octyldiphenylmethane, with an average of twenty ethylene oxide units per phenolic nucleus); the trimer D9a (also with twenty ethylene oxide units per nucleus); and the polymeric mixture D27 (twelve ethylene oxide units per nucleus). 'Triton A20' $50 \%$ (12.5\% effective agent), or the other products at $\mathbf{1 2 . 5} \%$, were autoclaved and, before use, diluted to $1.0 \%$. (All concentrations of 'Triton A20' given hereafter refer to the effective agent.)

Cultures. The strain of $M$. tuberculosis mainly used was H37 Ra, the avirulent variant of the human strain $\mathrm{H37}$; this, as well as the virulent variant H37 Rv and four additional avirulent human strains, R1Ra, JH6Ra, JH16Ra and H4Ra, were obtained from Dr W. Steenken, Jr., Trudeau Laboratory, New York (see Report, 1946; Steenken \& Gardner, 1946a, $b$; Yegian \& Kurung, 1952).

Media and tests. The avirulent strains of $M$. tuberculosis were maintained on Herrold's (1931) egg-yolk agar medium, containing $5 \%$ glycerol, and the virulent strain H37Rv on the surface of Proskauer \& Beck's defined medium containing asparagine (see Baldwin, Petroff \& Gardner, 1927). Well-grown cultures were ground in Griffith tubes to give aqueous suspensions equal in opacity (by eye) to a standard containing $\mathbf{0 \cdot 2} \mathbf{~ m g}$. (wet-weight) $/ \mathrm{ml}$. The liquid medium used for the repeated subculture and other tests had the following basal constituents: Difco Casamino acids, $2.5 \mathrm{~g}$.; asparagine, $0.3 \mathrm{~g}$.; anhydrous $\mathrm{Na}_{2} \mathrm{HPO}_{4}, 2 \cdot 5$ g.; $\mathrm{KH}_{2} \mathrm{PO}_{4}, 1 \cdot 0$ g.; sodium citrate, $1.5 \mathrm{~g}$.; $\mathrm{MgSO}_{4}, 7 \mathrm{H}_{2} \mathrm{O}, 0 \cdot 6 \mathrm{~g}$.; glycerol, $25 \mathrm{ml}$; d distilled water to $1000 \mathrm{ml}$. This basal medium was distributed in $5 \mathrm{ml}$. quantities in $25 \mathrm{~mm}$. diameter test tubes and autoclaved; and to each tube $0.5 \mathrm{ml} .5 \%$ albumin (bovine plasma fraction $\mathrm{V}$ ) sterilized by filtration, and a surface-active agent to give the required concentration, were added, the product being referred to hereafter as 'the liquid medium'. The inoculum, $0.2 \mathrm{ml}$. of a standard suspension $(0.04 \mathrm{mg}$. wet-weight bacilli) or 0.1-0.2 ml, of a culture grown in similar liquid medium, was then added, and the tubes incubated at $37^{\circ}$ for $2-3$ weeks. Alternatively, for in vivo experiments, the basal medium was distributed in $50 \mathrm{ml}$. quantities in $250 \mathrm{ml}$. conical flasks. The additions were then $5 \mathrm{ml} .5 \%$ albumin, a surface-active agent, and the inoculum ( $1 \mathrm{ml}$.) from a culture in a tube of similar medium; incubation was for 7-10 days (the flasks being shaken by circular motion twice daily to promote dispersion when a concentration of $0.001 \%$ 'Triton A20' was used).

Examination of cultures. The degree and character (granular or dispersed) of submerged growth in the liquid medium were determined by naked-eye, or with a hand-lens, after gentle shaking. The morphology was assessed by the microscopic examination, under $2 \mathrm{~mm}$. oil-immersion lens, of films prepared 
by placing loopfuls of the cultures on slides, fixing, and staining by the ZiehlNeelsen method.

Inoculation of mice. Cultures in the flasks (see above) were centrifuged, and the bacterial deposits re-suspended in 'Tween $80^{\prime}$ ' albumin + saline solution to give $1 \mathrm{mg}$. (wet-weight)/ml. (measured by a Spekker photo-electric absorptiometer). The bacillary suspensions, diluted in Tween + albumin + saline to contain the required doses in $0.5 \mathrm{ml}$, were injected into the tail veins of female mice (18-20 g.) of the albino $P$ strain. In one experiment the suspensions were prepared to standard visual opacity by grinding from egg-yolk agar cultures, and $0.5 \mathrm{ml} .(0.1 \mathrm{mg}$. wet-weight bacilli) used to inject by the same route.

\section{RESULTS}

Effect of repeated subculture in the presence of 'Triton A20' on the morphology of the avirulent strain $\mathrm{H} 37 \mathrm{Ra}$

Samples of suspensions prepared from egg-yolk agar cultures of H37 Ra were inoculated into tubes of the liquid medium containing 'Triton A 20' 0.001 or $\mathbf{0 . 0 0 2} \%$. After incubation, samples from these tubes were seeded into fresh tubes of medium containing the same concentrations of agent, and the process of transfer and culture repeated. These concentrations were chosen for three reasons: they had been effective in enhancing virulence of the VAL strain; microscopic 'cords' were more evident when VAL or H37 Rv was grown in these than in higher concentrations, such as $0.05 \%$; and in any case only low concentrations of 'Triton A 20' permit the growth of avirulent strains of M. tuberculosis (Rees \& Hart, 1952). Growth was slow and sparse but appeared to be continuous; it was submerged and was granular or partly dispersed. In a typical experiment, microscopic examination of the first culture in the medium containing $0.001 \%$ agent showed the bacilli arranged in characteristic amorphous clumps ( $\mathrm{Pl} .1$, fig. $1 a$ ), but a change was already evident after the second exposure during growth. The third serial culture showed semi-amorphous clumps of bacilli with some cord formation (Pl. 1, fig. $1 b$ ), while the tenth showed well-defined cords without any amorphous collections (Pl. 1, fig. 1c); this appearance was similar to that displayed by the virulent strain H37 Rv after one culture in the same medium (Pl. 1, fig. 2). Cord formation developed much less in the series grown in the presence of 'Triton A20' $0.002 \%$.

The degree and rate of change in morphology of H37 Ra varied considerably, and after cord formation had been induced, further transfers did not improve and might even reduce it. At the peak the cords were usually less tightly packed, less serpentine, and more branched, than those of H37Rv. Throughout the series the organisms remained strongly acid-fast.

To see whether prolonged growth would produce cords as well as repeated subculture, a first culture of H37 Ra in 'Triton A20' $0.001 \%$ was incubated for 7 weeks. Examination at 2 weeks showed the expected amorphous growth pattern; and there was no appreciable change at 7 weeks. 


\section{Stability of the microscopic cord pattern of growth of H37 Ra}

Stability of the cord-forming property was examined in two ways, using in each case tubes taken from a series of subcultures (ranging from the 6th to the 14th) in the liquid medium containing 'Triton A 20 ' $0.001 \%$ after the stage of well-defined cord formation had been reached. In the first method samples from such tubes were sown on to egg-yolk agar, and suspensions prepared from the resulting cultures were inoculated $(0.04 \mathrm{mg}$.) into tubes of the usual medium containing 'Triton A 20' $0.001,0.002$ or $0.05 \%$, as well as in the same medium without any agent (i.e. basal constituents plus albumin only). In the second method a sample from one such tube (11th in the series) was seeded ( $1 \mathrm{ml}$.) into a flask of the liquid medium containing 'Triton A 20' $0.001 \%$, or 0.2 and $0.5 \mathrm{mg}$. (wet-weight bacilli) of the resulting culture inoculated into two groups of six mice. One of the animals ( $0 \cdot 2 \mathrm{mg}$. group) died 32 days later with several large foci in the lungs; the other eleven were apparently well at 39 days, when they were killed, and pin-point pulmonary foci were found in the lungs of eight and more definite though limited lesions in those of three ( $0.2 \mathrm{mg}$. group). Cultures on to egg-yolk agar were made from the pulmonary lesions of the mouse that died and of one of those killed, after grinding with sand and treating the suspensions with acid; the cultures were tested precisely as in the first method.

The tests made with the egg-yolk agar cultures obtained by both these methods still showed a microscopic cord pattern of growth, not only in the presence of 'Triton A20' 0.001 or $0.002 \%$, but also in the liquid medium without any agent. The appearances after passage through mice to egg-yolk agar, and thence to the liquid medium devoid of the agent, are shown in Pl. 2, fig. $3 a$, and in the presence of the agent $0.001 \%$ in Pl. 2, fig. $3 b$. Some, though slow, growth of both sets of cultures also took place in the presence of 'Triton A 20' $0.05 \%$; this growth was dispersed but microscopically amorphous. Egg-yolk agar cultures of unmodified H37 Ra and of H37 Rv, inoculated into similar tubes for comparison, showed an amorphous growth pattern and well-marked cord formation, respectively, in the presence of 'Triton A 20' 0.001 or $0.002 \%$. In $0.05 \%$ of the agent the growth of the unmodified H37 Ra was inhibited (see above), while H37 Rv showed profuse short cords, as was to be expected.

When egg-yolk agar cultures of cord-forming H37 Ra were subcultured once again on egg-yolk agar before being inoculated into the liquid medium devoid of surface-active agent, partial reversion to the amorphous growth pattern was observed.

\section{Effect of the changed morphology on the inhibition of $\mathrm{H} 37 \mathrm{Ra}$ by the surface-active polyoxyethylene ether $\mathbf{D} 4$}

We have previously shown that one member of the series of surface-active polyoxyethylene ethers made in this Institute (the dimer D4) inhibited the growth of avirulent strains of $\boldsymbol{M}$. tuberculosis, whereas virulent, as well as attenuated, strains grew freely in its presence; a factor common to the strains 
naturally insusceptible, but absent from those inhibited, is the ability to produce some degree of microscopic cord pattern during growth (Rees \& Hart, 1952). We set out now to determine whether the induced production of cords by $\mathrm{H3} 7 \mathrm{Ra}$ was associated with a loss of susceptibility to $\mathrm{D} 4$. The observation, recorded above, that subcultures on egg-yolk agar of cord-forming organisms derived from $\mathrm{H37} \mathrm{Ra}$ would grow in moderate concentrations $(0.05 \%)$ of 'Triton A 20 ' (which mixture contains a significant amount of the dimer), whereas the original amorphous-growing $\mathrm{H37} R \mathrm{R}$ was inhibited by such concentrations, had given a hint of a change in susceptibility. Further use was made of these egg-yolk agar cultures (obtained from subcultures in the series in the medium containing 'Triton A20' 0.001\%) during the performance of the stability tests already described. Suspensions prepared from these cultures (whose cord-forming property persisted when grown in liquid medium containing 'Triton A 20' at low concentrations or in its absence, see above), and also from egg-yolk agar cultures of the unmodified H37 Ra, were tested simultaneously for growth inhibition by D4, using the usual liquid medium and comparable inocula $(0.04 \mathrm{mg}$.). The inhibitory end-point after 2 weeks' incubation was found to be $>\mathbf{0 . 1} \%$ for the cord-forming strains in contrast to $0.006 \%$ for the unmodified strain, though the growth of the former was sometimes first evident only during the second week. Microscopically, the cord-forming strain grew amorphously in $\mathrm{D} 4.0 .05 \%$, as in that concentration of 'Triton A 20', and the property of cord formation was much less striking in low concentrations of the dimer than of the latter agent.

\section{Effect of the concentration of 'Triton A 20 ' on cord formation}

The optimal concentration of 'Triton A20' for cord formation by H37 Ra after exposure during repeated subculture appeared to be about $0.001 \%$. Certainly $0.002 \%$ was less effective. Lower concentrations than $0.001 \%$ were not tested, but eight subcultures in liquid medium without any agent (i.e. containing albumin $0.5 \%$ alone) was unsuccessful in producing cords. As already stated, unmodified H37 Ra fails to grow in 'Triton A 20' $0.05 \%$, while cordforming strains, after transfer to egg-yolk agar, grow amorphously in this concentration. It was found, moreover, that such strains, when transferred from liquid medium containing 'Triton A20' $0.001 \%$ (in which they showed well-marked cords) directly into similar medium containing $0.05 \%$, also grew amorphously; but when the latter culture was inoculated (0.1 ml.) back into the medium but without agent (giving a final concentration again of $0.001 \%$ ), cords reappeared.

\section{Effect of repeated subculture in other surface-active agents}

The strain H37 Ra was subcultured repeatedly in liquid medium containing low concentrations of several members of our series of polyoxyethylene ethers. Cords developed in the presence of $0.001 \%$ D4 or D9a, and $0.002 \%$ D27, at the same rate as in 'Triton A 20'; the macroscopic character of the growth was similar. It should be mentioned also that on two occasions cultures of H37 Ra which had been transferred a number of times from and to the liquid 
medium containing 'Tween 80 ' $0.05 \%$ (the growth being amorphous, as would be expected), and which were then transferred to egg-yolk agar and finally to medium containing 'Triton A20', showed well-marked cord formation. These observations with 'Tween 80 ' were not reproducible at will.

Behaviour of other avirulent strains of M. tuberculosis

The four avirulent strains R1Ra, JH6Ra, JH16Ra and H4Ra were repeatedly subcultured in the presence of 'Triton $\mathrm{A} \mathrm{20}^{\text {' }}$ ' by the method used for H37 Ra. No clear evidence of cord formation was noted in any strain after at least seven exposures to the agent 0.001 or $0.002 \%$. The organisms of the H4Ra strain were strongly acid-fast, but those of the other three were largely acid-susceptible and remained so. In the case of $\mathrm{H} 4 \mathrm{Ra}$, although unsuccessful by serial culture, we have twice observed definite cord formation in the liquid medium containing this type of surface-active agent. The reproducibility of this phenomenon is being investigated.

\section{Virulence tests with the cord-forming strains}

Tubes from a series of subcultures (4th, 7th and 11th) of H37 Ra in the liquid medium containing 'Triton A20' $0.001 \%$, after the stage of cord formation had been reached, were used to inoculate flasks of the same medium and 0.001 or $0.002 \%$ of this agent; after growth, 0.2 and $0.5 \mathrm{mg}$. (wet-weight bacilli) of the cultures were used to infect mice. Only pin-point pulmonary lesions were seen when the animals were killed 40 days later, except in one group (11th subculture) where more definite lesions were present in some animals and in the only one that had died of the disease (p. 153). Even though egg-yolk agar cultures made from these apparently more significant lesions were still capable of forming cords in liquid medium, they produced no deaths and only pin-point lesions when used $(0.1 \mathrm{mg}$.) to infect a fresh group of mice.

Cytochemical test. Egg-yolk agar cultures of cord-forming and of unmodified H 37 Ra were used for the cytochemical test (Dubos \& Middlebrook, 1948). In neither case did the organisms take up the neutral red.

\section{Character of H37 Ra on Proskauer and Beck's medium}

Although we have, in general, maintained H37 Ra on egg-yolk agar, we are aware that the Trudeau Laboratory, which supplied our transplant, carries avirulent as well as virulent strains of tubercle bacilli on the surface of Proskauer and Beck's fluid medium, as a suitable medium for keeping these strains stable (Report, 1946). No increase of virulence in $\mathrm{H37} \mathrm{Ra}$ has been noted after the use of this method for many years (Dr W. Steenken, Jr., personal communication). We found that $\mathrm{H37} \mathrm{Ra}$, taken from egg-yolk agar and grown on Proskauer and Beck's medium, did not produce cords spontaneously in the pellicle, and was no more likely to produce cords on a first subculture from Proskauer and Beck's medium in our liquid medium containing 'Triton A 20' $0.001 \%$ than when a first subculture therein was made from egg-yolk agar. 


\section{DISCUSSION}

Although the arrangement of the bacilli in amorphous clumps appears to constitute the normal in vitro microscopic growth pattern of the standard avirulent strains used in the present investigation (Yegian \& Kurung, 1952), 'spontaneous' changes in morphology towards the formation of 'cords' have been occasionally noted previously. Yegian \& Kurung themselves mention one culture of $\mathrm{H} 37 \mathrm{Ra}$ which, after 3 years' maintenance in their laboratory in Tween + albumin medium, exhibited definite cord formation microscopically when cultivated in their test medium. The formation of cords has been regularly observed when $\mathbf{H 3 7} \mathbf{R a}$ is grown in oleic acid + albumin medium, or in Gey's balanced salt solution, to which chick embryo material has been added (Bloch, 1948; Friedmann \& Russell, 1953). The morphology reverts to the usual unoriented pattern of growth when the strain is returned to ordinary media devoid of this material.

The present findings with $\mathrm{H} 37 \mathrm{Ra}$ differ in three respects from those in media containing embryonic tissue. The medium is more clearly defined in composition, its active component appearing to be a synthetic non-ionic surface-active agent. Cords are not produced on first exposure but only after several subcultures in the effective medium. Once obtained, this property persists after transfer to medium from which the agent is absent, whether the transfer be direct or after passage through mice; after further subcultures, however, there is a tendency to reversion.

The methods reported here for inducing cord formation are empirical. That successful for $\mathrm{H37} \mathrm{Ra}$ is defined (though with inconstancy in the time of onset and quality of cords), but the few observations of cords in the strain H4Ra have not yet been systematized. Several non-ionic surface-active agents promote the change, and, if ' $T$ ween 80 ' be included, it is not attributable to any one chemical series. The conditions of cultivation for successful induction in $\mathrm{H37} \mathrm{Ra-low}$ concentrations of agent-are also optimal for manifesting the cords when their formation has been achieved. Yet the modification of morphology is not just due to the coincident presence of the surface-active agent, since it is still evident after return of the strain to the ordinary egg medium and subsequent subculture into the liquid medium also devoid of the agent.

The loss of the selective inhibitory effect of the dimer D4 on the growth of H37 Ra, when the latter comes to form cords in 'Triton A 20', is in keeping with our previous experience that the growth of any strain of tubercle bacillus which shows some degree of cord formation is relatively unaffected by $\mathrm{D} 4$ (Rees \& Hart, 1952). We had, however, previously found it easy to produce avirulent bacilli resistant to D4 or to 'Triton A 20' (which contains D4) without loss of the typical amorphous growth pattern, by subcultivating $\mathrm{H37} \mathrm{Ra}$ in increasing concentrations of these agents. The question therefore arises whether the loss of susceptibility to $\mathrm{D} 4$ in the present experiments is due to 'resistance' rather than to cord formation. This seems improbable because the exposures of $\mathrm{H37} \mathrm{Ra}$ during growth were to a constant low con- 


\section{Morphology of avirulent tubercle bacilli}

centration ( 0.001 or $0.002 \%$ ), and not to increasing strengths, of 'Triton A20'. Moreover, the growth of a culture of H37 Ra in which cord formation had been induced in the presence of 'Tween 80 ', whose basic structure is unrelated chemically to that of $\mathrm{D} 4$, was also found to be uninhibited by the latter.

Little information can be offered on whether the mechanism responsible for cord formation in an avirulent strain of $M$. tuberculosis is a selection of mutants or a direct 'adaptation'. An attractive hypothesis, suggested by the considerations in the previous paragraph, is that $\mathbf{D} 4$ or 'Triton $\mathbf{A 2 0}$ ', by inhibiting the growth of amorphous avirulent organisms and permitting the growth of cord-forming mutants, favours selection of the latter during serial subculture. This hypothesis might account for cord formation by H37 Ra in these two surface-active agents, though the facts that their effective concentrations are subinhibitory and that exposure of H37 Ra to increasing concentrations is more likely to give 'resistant' amorphous organisms than 'cord-formers' (see above) make it unlikely. The hypothesis could not, however, explain the phenomenon when it occurs in the presence of the trimer D9a, which has no growth-inhibitory action on avirulent or virulent bacilli (Rees \& Hart, 1952). Although this particular form of selection of mutants appears, therefore, to be excluded, mutation itself is not. In this connexion, the observation that prolonged incubation of a first culture of $\mathrm{H} 37 \mathrm{Ra}$ in the presence of a concentration of surface-active agent which is effective during repeated subculture fails to induce significant cord formation has some interest.

A broad correlation has been suggested between the morphology of mammalian tubercle bacilli and their virulence, namely, that when cultivated under specific conditions virulent strains form cords, avirulent strains grow amorphously, while attenuated strains present an intermediate pattern (Middlebrook, Dubos \& Pierce, 1947; Middlebrook, 1950). A more restricted definition of the relationship is given by Yegian \& Kurung (1952): 'while all cord-formers are not necessarily virulent, all virulent strains are cord-formers'. Nevertheless, the fact that cord formation can be induced in originally amorphous-growing standard avirulent strains makes it important to know whether virulence has appeared. Yegian \& Kurung observed gross lesions in the liver and spleen of a guinea-pig inoculated with their cord-forming culture of $\mathrm{H37} \mathrm{Ra}(\mathrm{H37} \mathrm{Ra}(\mathrm{x}))$, whereas a typical amorphous-growing culture gave no such lesions. Bloch found that when mice were given comparable doses of H37 Ra grown in medium containing chick embryo extract and in medium without this addition, neither group showed definite evidence of virulent infection by clinical deterioration or at autopsy. We have been unable to distinguish consistently the pathogenicity of cord-forming $\mathrm{Hz} 7 \mathrm{Ra}$ from that of unmodified $\mathrm{H} 37 \mathrm{Ra}$ in mice after heavy dosage, either on the basis of mortality or of gross pathology, even though cord-forming organisms could be recovered from the pulmonary lesions. This experience indicates that the correlation between virulence (as ordinarily measured) and cord formation is by no means close. Similar evidence is provided by strains with degraded virulence derived from $\mathrm{H} 37 \mathrm{Rv}$, which may show cords indistinguishable 
from those of the latter. On the other hand, these findings do not controvert the more restricted definition of the relationship between morphology and virulence referred to above.

In the cytochemical reaction, it is stated that virulent tubercle bacilli which form serpentine cords on growth, such as $\mathrm{H37} \mathrm{Rv}$, bind neutral red in alkaline medium, whereas avirulent strains such as $\mathrm{H37} \mathrm{Ra}$ do not (Dubos \& Middlebrook, 1948). Our finding that cord-forming $\mathrm{H37} \mathrm{Ra}$ behaved in the test like the unmodified strain may have little significance, since in our hands the differences in coloration between $\mathrm{H} 37 \mathrm{Ra}$ and $\mathrm{H37} \mathrm{Rv}$ have been only slight (cf. Richmond \& Cummings, 1950).

\section{REFERENCES}

Baldwin, E. R., Petroff, S. A. \& Gardner, L. U. (1927). Tuberculosis : Bacteriology, Pathology and Laboratory Diagnosis, p. 45. London: Baillière, Tindall and Cox.

BLOCH, H. (1948). The effect of chick embryo extract on the growth and morphology of tubercle bacilli. J. exp. Med. 88, 355.

Cornforth, J. W., Hart, P. D'A., Rees, R. J. W. \& Stock, J. A. (1951). Antituberculous effect of certain surface-active polyoxyethylene ethers in mice. Nature, Lond. 168, 150.

Dubos, R. J. \& Mrddlebrook, G. (1948). Cytochemical reaction of virulent tubercle bacilli. Amer. Rev. Tuberc. 58, 698.

Friedman, M. \& Byers, S. O. (1953). The mechanism responsible for the hypercholesteremia induced by 'Triton WR-1339'. J. exp. Med. 97, 117.

FriedmanN, I. \& Russell, K. (1953). Serpigenous growth of tubercle bacilli in media containing embryonic tissue. J. Path. Bact. 65, 495.

Hart, P. D'A., Long, D. A. \& Rees, R. J. W. (1952). Depression of tuberculin sensitivity in guinea-pigs by certain antituberculous surface-active agents. Brit. med. J. i, 680 .

Herrold, R. D. (1931). Egg-yolk agar medium for the growth of tubercle bacilli. J. infect. Dis. 48, 236.

Middlebrook, G. (1950). The experimental analysis of virulence of tubercle bacilli. Bull. N.Y. Acad. Med. 26, 498.

Middlebrook, G., Dubos, R. J. \& Pierce, C. (1947). Virulence and morphological characteristics of mammalian tubercle bacilli. J. exp. Med. 86, 175.

ReEs, R. J. W. (1953). Antituberculous activity of certain non-ionic detergents. Proc. roy. Soc. Med. 46, 581.

Rees, R. J. W. \& Hart, P. D'A. (1952). The inhibition of growth of avirulent strains of Mycobacterium tuberculosis by a surface-active polyoxyethylene ether. J.gen. Microbiol. 7, 372.

Report (1946). A depot for standard cultures of tubercle bacilli. Amer. Rev. Tuberc. $53,511$.

Richmond, L. \& Cummings, M. M. (1950). An evaluation of methods of testing the virulence of acid-fast bacilli. Amer. Rev. Tuberc. 62, 632.

Steenken, Jun., W. \& Gardner, L. U. (1946a). R1 strain of tubercle bacillus. Amer. Rev. Tuberc. 54, 51.

Steenken, Jun., W. \& Gardner, L. U. (1946b). History of H37 strain of tubercle bacillus. Amer. Rev. Tuberc. 54, 62.

Yegian, D. \& Kurung, J. (1952). Growth-pattern and virulence of tubercle bacilli. Amer. Rev. Tuberc. 65, 181. 
Journal of General Microbiology, Vol. 10, No. 1
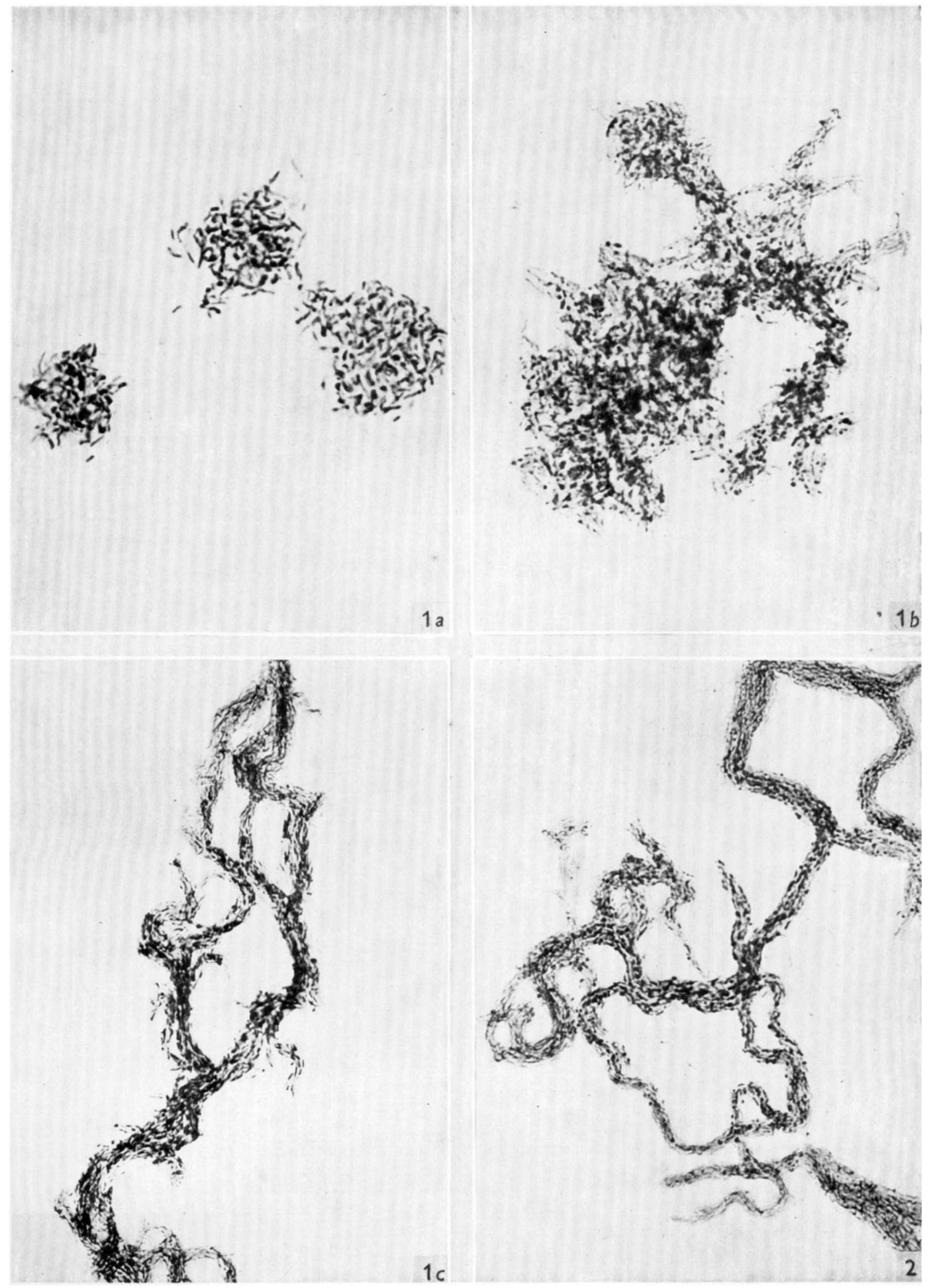

P. D'Arcy Hart \& R. J. W. Rhes-Morpilology of avirulent tublercle bacilit. Piate 1 
Journal of General Microbiology, Vol. 10, No. 1

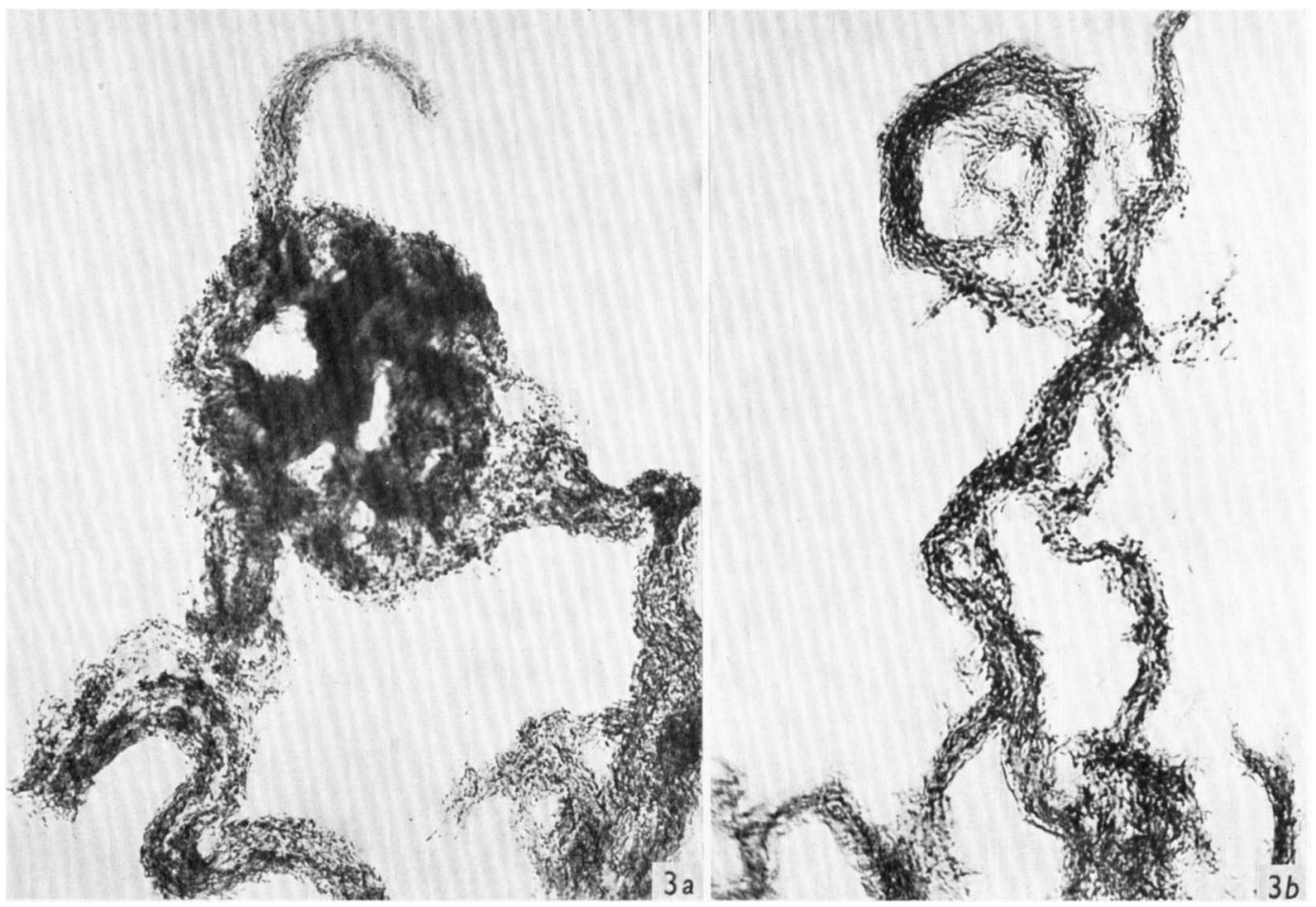

P. D'Arcy Har't \& R. J. W. Rees-Morphology of avirulen't tubercle: bacilit. Plate 2 


\section{EXPLANATION OF PLATES}

Plate 1

Fig. 1. a-c: Mycobacterium tuberculosis, avirulent strain H37 Ra, cultured repeatedly in the test liquid medium containing 'Triton A20' 0.001 \%. Ziehl-Neelsen stain. $a$, first serial culture, after 2 weeks' growth. Shows amorphous collections of bacilli. $\times 900$. $b$, third serial culture, after 2 weeks' growth. Shows semi-amorphous collections of bacilli, with commencing formation of cords. $\times 800$. $c$, ninth serial culture, after 2 weeks' growth. The bacilli are arranged in fairly well-developed cords. $\times 800$.

Fig. 2. Mycobacterium tuberculosis, virulent strain H37 Rv, after 2 weeks' growth in the test liquid medium containing 'Triton A20' 0.001\%. Shows well-developed 'tight' serpentine cords (compare with Fig. 1c). Ziehl-Neelsen stain. $\times 800$.

\section{Prate 2}

Fig. 3. $a, b$ : Cultures of organisms recovered, via egg-yolk agar, from the lungs of mice infected with cord-forming H37 Ra. Shows persistence of cord formation. ZiehlNeelsen stain. $a$, grown in the test liquid medium without surface-active agent. $\times 650$. $b$, grown in the test liquid medium containing 'Triton A20' $0.001 \% \cdot \times 800$. 